\title{
Zonas alvo de treino em diferentes ergómetros
}

César Chaves ${ }^{1}$, Rui Garganta ${ }^{1}$, Jorge Roig ${ }^{1}$

${ }^{1}$ IPVC, ESE, departamento de Motricidade Humana - Viana do Castelo, Minho, Portugal.

Chaves, C.; Garganta, R.; Roig, J.; Zonas alvo de treino em diferentes ergómetros.Motricidade 3(2): 9-12

\section{Introducão}

O exercício cardiovascular tem-se constituído como um dos meios fundamentais para a promoção da saúde e bem-estar das populações. As possibilidades para a sua aplicação são cada vez mais variadas, sobretudo nos ginásios, onde é usual o recurso a diversos tipos de ergómetros, seja na sala de cardiofitness ou nas aulas de grupo de cariz aeróbio. Neste sentido, uma ajuda fundamental é facultada pelos programas de treino, que, actualmente, permitem considerar os objectivos e as limitações de cada indivíduo, potenciando os benefícios do exercício e minimizando o tempo necessário para o alcance dos mesmos. Consequentemente, têm sido desenvolvidas a nível global, diversas linhas orientadoras para a realização da actividade fisica aeróbia. Instituições de referência, como o American College of Sports Medicine (ACSM), a American Heart Association (AHA), o Centers for Disease Control and Prevention (CDC), o Department of Human and Health Services (DHHS), o Institute of Medicine (IOM), o National Association for Sport and Physical Education (NASPE), o National Institutes of Health (NIH), o Surgeon General e o United States Dietary Guidelines (USDG), entre muitas outras, propõem várias directrizes, algumas das quais tendo inclusivamente em conta diferentes populações e patologias tentando, desta forma, personalizar e potenciar cada vez os programa de exercícios. De uma forma geral, estas instituições sugerem a exercitação numa dada percentagem de um valor de referência (frequência cardíaca máxima $-\mathrm{FC}_{\text {máx }}$ e/ou consumo máximo de oxigénio - $\mathrm{VO}_{2}$ máx), variável em função do objectivo pretendido. Estes intervalos percentuais, vulgarmente designados por "zonas alvo de treino", são ainda baseados em função do limiar de lactato ou ventilatório. Todavia, esta estimativa é efectuada de forma indiferenciada, não contemplando as contingências específicas de cada ergómetro ou modo de exercício, que se sabe serem capazes de condicionar o comportamento das respostas fisiológicas e, assim, não corresponder aos objectivos de quem o pratica. Objectivo: identificar e comparar os valores da $\mathrm{FC}_{\text {máx }}$, do $\mathrm{VO}_{2}$ máx e do limiar ventilatório (LV), em quatro ergómetros utilizados para o treino cardiovascular.

\section{Metodologia}

A amostra é constituída por 6 sujeitos adultos com idades médias de $30 \pm 8$ anos, fisicamente activos e aparentemente saudáveis. Todos os sujeitos realizaram testes de esforço máximo em condições estandardizadas, nos ergómetros testados (tapete rolante "Stex", modelo "7020 T"; bicicleta "SciFit", modelo "Iso 1000"; elíptica "Pulse", modelo "280 F"; manivela "SciFit", modelo "Pro 1000”), não sendo especializados em nenhum deles. A duração de todos os testes foi definida em 10 minutos e a sua maximalidade confirmou-se pela constatação de um platô na curva do consumo de oxigénio, um quociente respiratório superior a 1,10 , percepção subjectiva máxima de esforço através da escala de Borg adaptada e concentração de lactato sanguíneo pós-esforço superior a $8 \mathrm{mmol} / \mathrm{l}$. As variáveis em análise foram a frequência cardíaca máxima $\left(\mathrm{FC}_{\text {maxx }}\right)$, a frequência cardíaca ao limiar ventilatório (FCLim), (com recurso a um cárdio-frequencímetro da marca "Polar"), o consumo máximo de oxigénio ( $\mathrm{VO}_{2}$ máx) e o consumo de oxigénio ao limiar ventilatório $\left(\mathrm{VO}_{2} \mathrm{Lim}\right)$, (através do oxímetro "Cortex", modelo "2000", com registos de respiração a respiração). A análise das diferenças entre os indicadores avaliados, foi efectuada a partir da ANOVA (Analysis of Variance) de medi- 


\begin{tabular}{|c|c|c|c|c|c|c|c|c|c|c|c|c|}
\hline \multicolumn{13}{|c|}{$\begin{array}{l}\text { Quadro 1: Comparação das } \mathrm{FC}_{\text {máx }} \mathrm{E} \\
\text { cardíacas máximas teóricas - atrav } \\
\text { e a diferença entre as duas (dif.). }\end{array}$} \\
\hline \multirow[b]{3}{*}{ a } & \multicolumn{3}{|c|}{ Tapete } & \multicolumn{3}{|c|}{ Elíptica } & \multicolumn{3}{|c|}{ Bicicleta } & \multicolumn{3}{|c|}{ Manivela } \\
\hline & $\mathrm{FC}_{\text {máx }} \mathrm{E}$ & $\mathrm{FC}_{\text {máx }} \mathrm{T}^{-}$ & |dif.| & $\mathrm{FC}_{\text {máx }} \mathrm{E}$ & $\mathrm{FC}_{\text {máx }} \mathrm{T}^{-}$ & |dif.| & $\mathrm{FC}_{\text {máx }} \mathrm{E}$ & $\mathrm{FC}_{\text {máx }} \mathrm{T}^{-}$ & |dif.| & $\mathrm{FC}_{\text {máx }} \mathrm{E}$ & $\mathrm{FC}_{\text {máx }} \mathrm{T}$ & |dif.| \\
\hline & 189 & 197 & $|8|$ & 183 & 197 & $|14|$ & 178 & 197 & $|19|$ & 170 & 197 & |27| \\
\hline b & 192 & 176 & $|16|$ & 180 & 176 & $|4|$ & 185 & 176 & $|9|$ & 173 & 176 & $|3|$ \\
\hline c & 191 & 188 & $|3|$ & 180 & 188 & $|8|$ & 180 & 188 & $|8|$ & 159 & 188 & $|29|$ \\
\hline d & 194 & 188 & $|6|$ & 191 & 188 & $|3|$ & 185 & 188 & $|3|$ & 182 & 188 & $|6|$ \\
\hline e & 193 & 197 & $|4|$ & 187 & 197 & $|10|$ & 171 & 197 & $|26|$ & 180 & 197 & $|17|$ \\
\hline$f$ & 211 & 194 & $|17|$ & 204 & 194 & $|10|$ & 188 & 194 & $|6|$ & 194 & 194 & $|0|$ \\
\hline
\end{tabular}

\begin{tabular}{ccccc}
\hline \multicolumn{2}{l}{ Quadro 2: Resumo dos resultados encontrados das diferentes variáveis para cada ergómetro. } \\
\hline Tapete & $\mathrm{FC}_{\text {máx }}$ & FCLim & VO $_{2}$ máx & VO $_{2}$ Lim \\
Elíptica & 195 & 184 & 52 & 48 \\
Bicicleta & 188 & 165 & 52 & 42 \\
Manivela & 181 & 161 & 46 & 37 \\
\hline
\end{tabular}

das repetidas, recorrendo ao teste multivariado com a sugestão de Lambda de Wilks. No caso de se registarem diferenças com significado estatístico, as múltiplas comparações foram realizadas através do "teste de Bonferroni". O nível de significância foi mantido em 0,05 .

\section{Resultados}

Obtidos sugerem que: a) de uma forma geral, os valores alcançados nas variáveis em análise, nos diferentes ergómetros, são substantivamente distintos (quadros 1 e 2). Os mais elevados são geralmente obtidos no tapete rolante, seguido 워․ da elíptica, do ciclo-ergómetro e, por último, . da manivela. É possível detectar diferenças com significado estatístico entre o tapete e todos o orgómetros e entre a elíptica e a manivela (quanto à $\mathrm{FC}_{\text {máx }}$ e à FCLim), entre manivela e todos os ergómetros $\left(\mathrm{VO}_{2}\right.$ máx $)$ e entre a manivela e o tapete e elíptica, e entre tapete e bicicleta ( $\left.\mathrm{VO}_{2} \mathrm{Lim}\right)$.

b) A utilização de fórmulas teóricas (neste caso “220-idade") para a predição da $\mathrm{FC}_{\text {máx }}$ deve ser evitada, dado o seu erro significativo em relação à $\mathrm{FC}_{\text {máx }} \mathrm{E}$ (quadro 1). Pelos pontos anteriores se conclui que as zonas alvo de treino deverão ser necessariamente distintas, para cada ergómetro, ainda que o objectivo se mantenha o mesmo. Sugestões: para a aplicação dos resultados obtidos (com base na FC): Para uma correcta prescrição do exercício em diferentes ergómetros, 
Zonas alvo de treino em diferentes ergómetros

César Chaves, Rui Garganta, Jorge Roig

Quadro 3: Estimativa da correspondência entre as frequências cardíacas máximas obtidas nos vários ergómetros.

\begin{tabular}{|c|c|c|c|}
\hline & Elíptica & Bicicleta & Manivela \\
\hline Tapete & $96 \%$ & $93 \%$ & $90 \%$ \\
\hline Elíptica & & $96 \%$ & $94 \%$ \\
\hline Bicicleta & & & $97 \%$ \\
\hline \multicolumn{4}{|c|}{$\begin{array}{l}\text { Quadro 4: Percentagem da frequência cardíaca máxima a que ocorre o limiar ventilatório em cada ergómetro } \\
\text { (PerFC }{ }_{\text {máx }} \text {. }\end{array}$} \\
\hline \multicolumn{4}{|c|}{ PerFC $_{\text {máx }}$} \\
\hline Tapete & \multicolumn{3}{|c|}{94} \\
\hline Elíptica & \multicolumn{3}{|c|}{88} \\
\hline Bicicleta & \multicolumn{3}{|c|}{89} \\
\hline Manivela & \multicolumn{3}{|c|}{86} \\
\hline
\end{tabular}

sugerimos que: 1) se determine as $\mathrm{FC}_{\text {máx }}$ alcançadas em cada ergómetro, ou apenas num deles, sendo as outras extrapoladas através da consulta do quadro 3; e 2) se determine a percentagem da $\mathrm{FC}_{\text {máx }}$ a que ocorre o LV em cada ergómetro, pela consulta do quadro 4.

\section{Conclusões}

As recomendações para a prescrição do exercício e respectivas zonas alvo de treino, deverão considerar cada ergómetro ou modo de exercício, atendendo a que as $\mathrm{FC}_{\text {máx }}$ e/ou o $\mathrm{VO}_{2}$ máx e o momento de ocorrência do LV são distintos entre a maioria dos ergómetros, condicionando a efectividade do treino.

\section{Correspondência}

César Chaves

cesarchaves@ese.ipvc.pt 